\title{
Occurrence and antimicrobial susceptibility of enteric rods and pseudomonads isolated from the dental prostheses biofilm
}

Sanrrangers Sales SILVA', Maximilo de Oliveira RIBEIRO ${ }^{2}$, Francisco Isaac Fernandes GOMES', Hellíada Vasconcelos CHAVES ${ }^{1}$, Antonio Alfredo Rodrigues e SILVA ${ }^{3}$, Iriana Carla Junqueira ZANIN ${ }^{1}$, Francisco Cesar Barroso BARBOSA ${ }^{1}$

\footnotetext{
1- Universidade Federal do Ceará, Faculdade de Odontologia, Campus Sobral, Sobral, CE, Brasil.

2- Universidade Federal do Ceará, Faculdade de Medicina, Campus Sobral,Sobral, CE, Brasil.

3- Agência Nacional de Vigilância Sanitária, Brasília, DF, Brasil.
}

Corresponding address: Francisco Cesar Barroso Barbosa - Faculdade de Medicina de Sobral - Universidade Federal do Ceará - Avenida Comandante Maurocélio Rocha Ponte, 100 - Derby - Sobral - CE - Brazil - 62-042-280 - Phone: +55 (88) 3611.2202 - E-mail: fcbbarbosa@yahoo.com.br

Submitted: February 10, 2016 - Modification: June 01, 2016 - Accepted: June 16, 2016

\section{ABSTRACT}

\begin{abstract}
spiration of oral bacteria leads to cardiac and respiratory infectious diseases and dentures can act as a reservoir for pathogenic microorganisms. Objective: To determine the occurrence and the in vitro antimicrobial susceptibility of enteric rods and pseudomonads from the denture biofilm of 52 subjects at the Center for Dental Specialties of Sobral/ Ceará, Brazil. Material and Methods: Denture biofilm was collected and samples plated on MacConkey agar. The isolated bacterial colonies were identified using the BBL Crystal enteric/non-fermenter system. Antibiotic bacterial susceptibility was assessed by the disc diffusion method of amoxicillin, amoxicillin/clavulanic acid, doxycycline, tetracycline, tobramycin, imipenem, cefotaxime, and ciprofloxacin. The Minimum Inhibitory Concentration (MIC) of cefotaxime, tobramycin, doxycycline, imipenem, and ciprofloxacin was determined for 40 species by E-test. Results: 34 subjects (65.4\%) harbored enteric rods in their prostheses. Klebsiella pneumoniae (26.5\%), Escherichia coli $(23.5 \%)$, and Enterobacter aerogenes $(23.5 \%)$ were the most prevalent species. All organisms were susceptible to ciprofloxacin and most species were resistant to amoxicillin or amoxicillin/clavulanic acid, demonstrating variable sensitivity patterns to other antimicrobials. However, the MIC showed the emergence of strains with reduced sensitivity to ciprofloxacin (MIC ${ }_{90} \geq 3 \mu \mathrm{g} /$ $\mathrm{mL}$ ) and cefotaxime ( $\mathrm{MIC}_{90} \geq 2 \mu \mathrm{g} / \mathrm{mL}$ ). Conclusion: The findings show high prevalence of nosocomial diseases-related bacterial species and low susceptibility to antimicrobial drugs. Therefore, these results imply caution against the indiscriminate use of broad spectrum antibiotics in dental practice.
\end{abstract}

Keywords: Biofilms. Enterobacteriaceae. Pseudomonas. Drug resistance.

\section{NTRODUCTI ON}

Variations in the oral microbiota are directly related to age and have been attributed to the use of dentures ${ }^{16}$. The oral health status declines as a result of the aging process and individuals culminate with the need of dental prostheses, affecting their health, functional activities, and self-esteem ${ }^{8}$. Worldwide, 810 million people are aged 60 or over, which is predicted to increase to at least two billion by $2050,22 \%$ of the entire global population, and this demographic change will result in significant challenges for oral health care delivery, to an increasingly aged population with declining oral health ${ }^{13,19,31}$.

These individuals may not be able to clean their dentures properly, which in turn could result in the formation of a potentially pathogenic biofilm, exposing these individuals to the possibility of systemic severe infections. Currently, denture liners are available as silicone-based and acrylic resinbased. The adhesion to these materials depends on the properties of the surface of the microbial cells that will adhere and form biofilm, thus forming a 
complex three-dimensional architecture. One of the problems directly related to these materials is still the accumulation of biofilm on dentures, which in turn has received little attention by patients and general clinicians as its dental counterpart ${ }^{32}$.

The presence of enteric rods and pseudomonads in the denture biofilm should be highlighted because of their pathogenic potential and ability to adhere to solid surfaces ${ }^{2,17}$. In addition, infections caused by these organisms can be difficult to treat as a result of the bacterial resistance to a variety of antibiotics, including $\beta$-lactams, amoxicillin/clavulanic acid, cephalosporins, aminoglycosides, carbapenems, chloramphenicol, aztreonam, trimethoprim/ sulfametaxazol, tetracycline, and doxycycline 5,24.

The clinical importance of the non-fermenters gram-negative bacilli presence in the denture biofilm has significantly increased because of infections, high rates of morbidity, mortality in hospitalized subjects, and high levels of resistance. The dissemination of gram-negative bacteria with acquired antimicrobial drugs resistance is becoming a global problem. The propagation and dissemination of these microorganisms have already been confirmed ${ }^{11,25}$.

Enteric microorganisms and pseudomonads have demonstrated significant levels of resistance for all $\beta$-lactams, except for imipenem and meropenem. However, there are current reports of resistant enteric rods to carbapenems ${ }^{30}$. Among bacterial isolates resistant to ampicillin or amoxicillin, the most resistant were $\beta$-lactamase producers. The production of these hydrolytic enzymes seems to be the major mechanism of resistance to $\beta$-lactams, excluding most pseudomonads, in which $\beta$-lactamases were not detected. On the other hand, resistance to tetracycline was widely disseminated in the microbial enteric rods, and many of tested microorganisms were resistant ${ }^{25}$.

Having known such information, our hypotheses are that wearing dental prostheses older people may harbor superinfecting microorganisms in their oral cavity as a result of the biofilm formation on the surface of these appliances as well as these bacterial species could possess antimicrobial resistance. Therefore, we consider relevant to determine the prevalence of Enterobacteriaceae and Pseudomonadaceae species isolated from the denture biofilm and to assess their in vitro susceptibility to antimicrobial drugs, since these opportunistic microorganisms were previously reported to be on the dental prostheses biofilm ${ }^{15,28,29}$.

\section{MATERI AL AND METHODS}

\section{Study population}

Enteric microorganisms were isolated from the inner surface of dentures of 52 subjects within a 2-year follow-up period (2007-2008) at the Center for Dental Specialties - Sobral, State of Ceará, Brazil. The requirements for inclusion in this study were: no history of diabetes, no use of antimicrobials in the past three months, nor other medication that could affect their systemic or local immune system. All subjects signed a written consent form that was approved by the Institutional Review Board of Federal University of Ceará (COMEPE no 258/07).

\section{Data collection}

All subjects answered a questionnaire about their systemic health and a dental and soft tissue examination was performed to assess their oral conditions. The resulting findings were recorded in the medical record of each patient. Initially, the surfaces of the dentures were thoroughly dried with sterilized gauze to avoid contamination by saliva. The biofilm on the inner surface of complete dentures was collected with the aid of a sterile swab, and the samples were immediately placed in sterile phosphate buffered saline (PBS, pH 7.5, $0.8 \% \mathrm{NaCl}$ ) to the Department of Microbiology and Parasitology, Federal University of Ceará, in Sobral, and processed within a maximum of 2 hours after collection. Samples were dispersed by agitation (30 seconds) and serially diluted $\left(10^{-1}\right.$ and $\left.10^{-2}\right)$ in PBS. Aliquots of $100 \mu \mathrm{L}$ of the solutions obtained were plated on MacConkey agar (Acumedia, Lansing, Michigan, USA). Then, the plates were incubated at $36^{\circ} \mathrm{C} \pm 1^{\circ} \mathrm{C}$ for 24 hours.

\section{Microorganisms and microbial identification}

The isolated bacterial colonies were identified by Gram staining, colony morphology on MacConkey agar plates (Acumedia, Lansing, Michigan, USA), production of oxidase (Oxidase Newprov Strips, Pinhais, SP, Brazil), and biochemical identification kits (Kit for Identification of Enterobacteriaceae Newprov). The definitive identification of glucose enteric bacilli fermenters, oxidase-negative bacilli, and non-fermenter bacilli was carried out by the system BBL Crystal Enteric/Nonfermenter (Becton Dickinson Systems, Cockeysville, Maryland, USA) according to the manufacturer's instructions. The sample Escherichia coli ATCC 25922 was used as control.

\section{Antimicrobial susceptibility test}

The identified and isolated bacterial colonies previously collected were stored in broth heart infusion medium (Acumedia, Lansing, Michigan, USA) with glycerol $(3: 1)$ at $-80^{\circ} \mathrm{C}$ before performing the antimicrobial susceptibility tests. Then, each strain was seeded in broth heart infusion medium (Acumedia, Lansing, Michigan, USA) and incubated in a microbiological greenhouse at $36^{\circ} \mathrm{C} \pm 1^{\circ} \mathrm{C}$ for 24 
hours. Thereafter, in order to perform the sensitivity test, it was used the disk diffusion method of the Clinical \& Laboratory Standards Institute manual ${ }^{6}$. The antimicrobial drugs tested were amoxicillin (10 $\mu \mathrm{g})$, amoxicillin/clavulanate $(20 / 10 \mu \mathrm{g})$, doxycycline $(30 \mu \mathrm{g})$, tetracycline $(30 \mu \mathrm{g})$, tobramycin (10 $\mu \mathrm{g})$, imipenem $(10 \mu \mathrm{g})$, cefotaxime $(30 \mu \mathrm{g})$, and ciprofloxacin $(5 \mu \mathrm{g})$. Mueller Hinton agar was the medium used for this test. After seeding and incubating the samples in a microbiological greenhouse at $36^{\circ} \mathrm{C} \pm 1^{\circ} \mathrm{C}$ for 24 hours, the zones of inhibition were measured and the organisms were classified as sensitive, intermediate, or resistant according to CLSI references ${ }^{6,7}$. A total of 52 strains of enteric rods and pseudomonads were submitted to susceptibility tests. For multidrug resistant species, the Minimum Inhibitory Concentration (MIC) was determined for the following drugs: cefotaxime (CT), tobramycin (TM), doxycycline (DC), imipenem (IP), and ciprofloxacin (CI); the methodology used was E-test ${ }^{\circledR}$ (AB Biodisk, Solna, Sweden) and interpretations were made according to the CLSI references ${ }^{6,7}$.

\section{Statistical analysis}

The Mann-Whitney test was used to determine differences regarding age groups for men and women, and the chi-square test was used to access differences between genders, age groups, and time of prostheses use correlated between the presence and absence of the studied microorganisms. Differences of $p<0.05$ were considered statistically significant.

\section{RESULTS}

The population consisted of 52 individuals, of whom 42 (80.8\%) were women, ranging from 35 to 81 years old $(61.7 \pm 10.6)$, and $10(19.2 \%)$ were men, ranging from 53 to 93 years old ( $72 \pm 13.2$ ). There was no statistically significant difference between men and women regarding age groups $(p=0.245)$ and the average time of denture usage was around 11.09 years ( \pm 9.6$)$ (Table 1$)$. Regarding their systemic health status, 31 (59.6\%) subjects reported to have systemic hypertension, five (9.6\%) reported to be diagnosed with type 2 diabetes, three $(5.7 \%)$ reported to have gastritis, one $(1.9 \%)$ reported to be asthmatic, and one $(1.9 \%)$ to have osteoporosis, while 11 (21.1\%) were systemically healthy at anamnesis. Regarding their oral condition, we diagnosed three (5.7\%) subjects with candidiasis, two (3.8\%) with stomatitis, one $(1.9 \%)$ with xerostomia, while $46(88.4 \%)$ were found to be orally healthy at anamnesis.

According to Tables 2 and 3, 34 (65.4\%) subjects harbored enteric bacilli and/or pseudomonas species on dental prostheses. Most of these subjects (91.2\%) aged over 50. Also, the studied microorganisms were found in $15(75 \%)$ subjects in the group of subjects who wore dental prostheses over a 10-year period of time (Table 4). However, there was no statistically significant difference between the time of prostheses usage and the presence or absence of microorganisms ( $p>0.05)$.

Gender did not influence the colonization of the prosthesis, since no significant difference

Table 1- Age and gender distribution of subjects in the 2007-2008 period

\begin{tabular}{cccccccc}
\hline AGE & & MEN & \multicolumn{2}{c}{ WOMEN } & \multicolumn{2}{c}{ TOTAL } \\
\hline $35-49$ & $\mathbf{n}$ & $\%$ & $\mathbf{n}$ & $\%$ & $\mathbf{n}$ & $\%$ \\
\hline $50-64$ & 0 & 00.0 & 4 & 9.5 & 4 & 9.5 \\
$65-79$ & 3 & 30.0 & 19 & 45.2 & 22 & 42.3 \\
$\geq 80$ & 5 & 50.0 & 17 & 40.5 & 22 & 42.3 \\
Total & 2 & 20.0 & 2 & 4.8 & 4 & 7.7 \\
\hline
\end{tabular}

Table 2- Bacterial species distribution isolated from dental prostheses biofilm

\begin{tabular}{lcccc}
\hline \multicolumn{1}{c}{ MICROORGANISM } & MEN & \multicolumn{2}{c}{ WOMEN } & n \\
\hline Only enteric bacilli & $\mathbf{n}$ & $\mathbf{\%}$ & 24 & 57.1 \\
Only Pseudomonadaceae & 6 & 60.0 & 1 & 2.4 \\
Enteric bacilli and Pseudomonadaceae & 0 & 00.0 & 1 & 2.4 \\
Other species of microrganisms & 0 & 00.0 & 2 & 4.8 \\
Absence of microrganisms & 0 & 00.0 & 14 & 33.3 \\
Total of individuals & 4 & 40.0 & 42 & 100.0 \\
\hline
\end{tabular}


was observed in this study $(p=0.74)$. Across the 42-woman group, 24 (57.1\%) had their dentures solely contaminated with enteric bacilli, one participant (2.4\%) had Pseudomonas species, and another one $(2.4 \%)$ harbored enteric bacilli and pseudomonads. Furthermore, an 81-year old participant, who wore prostheses for 1 year, and a 59-year old subject, exceeding 20 years of denture use, had Acinetobacter iwoffi and Burkholderia pseudomallei detected on the biofilm from their dental prostheses.

Regarding the species found, Klebsiella pneumoniae, Escherichia coli, and Enterobacter aerogenes were the bacteria most prevalent detected, being isolated from dentures of nine (26.5\%), eight $(23.5 \%)$, and eight $(23.5 \%)$ individuals, respectively. It was also detected Citrobacter freundii (11.8\%), Klebsiella ozaenae $(11.8 \%)$, Klebsiella oxytoca $(8.8 \%)$, Enterobacter cloacae $(8.8 \%)$, Serratia marcescens $(8.8 \%)$, Serratia liquefaciens (8.8\%), Enterobacter gergoviae (5.9\%), Pseudomonas aeruginosa (5.9\%), Pseudomonas putida (2.9\%), Enterobacter sakasakii (2.9\%), Burkholderia pseudomallei (2.9\%), and Acinetobacter iwoffi (2.9\%).

Table 5 lists the antimicrobial susceptibility of all detected bacterial species. All strains were susceptible to ciprofloxacin and imipenem, except one of $E$. coli. Most species were resistant to amoxicillin or amoxicillin associated with clavulanic acid. The results from the MIC test on the bacterial species showed multidrug resistance patterns (Table 6 ): $33.4 \%$ of the $K$. pneumoniae species were resistant to cefotaxime (CT) and $22.2 \%$ to doxycycline (DC), being $11.1 \%$ of these organisms resistant to the other analyzed antimicrobial drugs.

Moreover, $37.5 \%$ and $12.5 \%$ of $E$. coli strains were resistant to doxycycline and tobramycin (TM), respectively, while $12.5 \%$ of Enterobacter aerogenes were resistant to the same antibiotics. In addition, $25 \%$ of species of Citrobacter freundii were resistant to doxycycline and imipenem (IP) and $33.3 \%$ of $E$. cloacae demonstrated insensitivity to doxycycline. The Serratia marcescens strains showed resistance to cefotaxime and to tobramycin. The two (100\%) Serratia liquefaciens strains and

Table 3- Number of analyzed subjects with dental prostheses harboring enteric rods, Pseudomonas, Acinetobacter spp., and Burkholderia spp. associated with groups of age and gender

\begin{tabular}{cccccccc}
\hline AGE & \multicolumn{2}{c}{ MEN } & \multicolumn{2}{c}{ WOMEN } & \multicolumn{3}{c}{ TOTAL } \\
& $\mathbf{h} / \mathbf{n}$ & $\mathbf{h} \%$ & $\mathbf{h} / \mathbf{n}$ & $\mathbf{h} \%$ & $\mathbf{h} \mathbf{n}$ & $\mathbf{h} \%$ \\
\hline $35-49$ & $0 / 0$ & 0.0 & $3 / 4$ & 75.0 & $3 / 4$ & 75.0 \\
$50-64$ & $2 / 3$ & 66.7 & $11 / 19$ & 57.9 & $13 / 22$ & 59.1 \\
$65-79$ & $3 / 5$ & 60.0 & $12 / 17$ & 70.6 & $15 / 22$ & 68.2 \\
$\geq 80$ & $1 / 2$ & 50.0 & $2 / 2$ & 100.0 & $3 / 4$ & 75.0 \\
All ages & $6 / 10$ & 60.0 & $28 / 42$ & 66.7 & $34 / 52$ & 65.4 \\
\hline
\end{tabular}

$\mathrm{n}=$ subjects in each group

$\mathrm{h}=$ harboring subjects in each group

$\mathrm{h} \%=$ percentage of harboring subjects

Table 4- Number of subjects wearing harbored or non-harbored dental prostheses by enteric rods, Pseudomonas, Acinetobacter spp., and Burkholderia spp. associated for time of dental prostheses usage

\begin{tabular}{ccccc}
\hline $\begin{array}{c}\text { TIME OF USAGE } \\
\text { (YEARS) }\end{array}$ & \multicolumn{2}{c}{ CONTAMINATED } & \multicolumn{2}{c}{ CONTAMINATED } \\
\hline $0-10$ & $19 / \mathbf{n}$ & $\mathbf{h} \%$ & $\mathbf{n h} / \mathbf{n}$ & $\mathbf{n h} \%$ \\
\hline $11-20$ & $11 / 13$ & 59.4 & $13 / 32$ & 40.6 \\
$21-30$ & $4 / 6$ & 84.6 & $2 / 13$ & 15.4 \\
$31-40$ & $0 / 1$ & 66.6 & $2 / 6$ & 33.4 \\
\hline
\end{tabular}

$\mathrm{n}=$ subjects in each group

$\mathrm{h}=$ harboring subjects in each group

$\mathrm{h} \%=$ percentage of harboring subjects

$\mathrm{nh}=$ non-harboring subjects in each group

$\mathrm{nh} \%=$ percentage of non-harboring subjects 
Table 5- Antimicrobial susceptibility of microorganisms isolated from denture biofilms

\begin{tabular}{|c|c|c|c|c|c|c|c|c|}
\hline Microorganism & CIP & IPM & TET & CTX & AMO & DOX & AMC & ТОВ \\
\hline Klebsiella pneumoniae (9) & $S(9)$ & $S(9)$ & $\begin{array}{c}\mathrm{S}(3) \mathrm{I}(1) \\
\mathrm{R}(5)\end{array}$ & $\begin{array}{c}S(7) !(1) \\
R(1)\end{array}$ & $R(9)$ & $\begin{array}{c}S(4) !(3) \\
R(2)\end{array}$ & $\begin{array}{c}S(4) !(1) \\
R(4)\end{array}$ & $\mathrm{S}(8) \mathrm{R}(1)$ \\
\hline Escherichia coli (8) & $S(8)$ & $S(7) R(1)$ & $S(5) R(3)$ & $S(6) R(2)$ & $\mathrm{S}(3) \mathrm{R}(5)$ & $\begin{array}{c}S(6) !(1) \\
R(1)\end{array}$ & $\begin{array}{c}\mathrm{S}(3) !(1) \\
\mathrm{R}(4)\end{array}$ & $S(7) R(1)$ \\
\hline Enterobacter aerogenes (8) & $S(8)$ & $S(8)$ & $S(6) R(2)$ & $S(7) \mid(1)$ & $\mathrm{R}(8)$ & $S(6) R(2)$ & $\mathrm{R}(8)$ & $S(8)$ \\
\hline Citrobacter freundii (4) & $S(4)$ & $S(4)$ & $S(3) R(1)$ & $\begin{array}{c}S(2) !(1) \\
R(1)\end{array}$ & $\mathrm{I}(1) \mathrm{R}(3)$ & $\begin{array}{c}S(2) !(1) \\
R(1)\end{array}$ & $\begin{array}{c}S(1) !(2) \\
R(1)\end{array}$ & $S(4)$ \\
\hline Klebsiella ozaenae (4) & $S(4)$ & $S(4)$ & $S(4)$ & $\mathrm{S}(4)$ & $S(1) R(3)$ & $S(4)$ & $S(4)$ & $S(4)$ \\
\hline Klebsiella oxytoca (3) & $S(3)$ & $S(3)$ & $S(3)$ & $S(2) R(1)$ & $\mathrm{R}(3)$ & $S(3)$ & $S(2) R(1)$ & $S(3)$ \\
\hline Enterobacter cloacae (3) & $S(3)$ & $S(3)$ & $\mathrm{I}(1) \mathrm{R}(2)$ & $\begin{array}{c}S(1) !(1) \\
R(1)\end{array}$ & $R(3)$ & $S(1) R(2)$ & $\mathrm{I}(1) \mathrm{R}(2)$ & $S(2) R(1)$ \\
\hline Serratia marcescens (3) & $S(3)$ & $S(3)$ & $S(2) R(1)$ & $S(2) !(1)$ & $S(1) R(2)$ & $S(3)$ & $S(1) R(2)$ & $S(2) R(1)$ \\
\hline Serratia liquefaciens (2) & $S(2)$ & $S(2)$ & $\mathrm{I}(2)$ & $\mathrm{I}(1) \mathrm{R}(1)$ & $\mathrm{R}(2)$ & $S(1) R(1)$ & $\mathrm{R}(2)$ & $S(2)$ \\
\hline Enterobacter gergoviae (2) & $S(2)$ & $S(2)$ & $S(1) !(1)$ & $S(1) \mid(1)$ & $\mathrm{R}(2)$ & $S(1) R(1)$ & $\mathrm{R}(2)$ & $S(2)$ \\
\hline Pseudomonas aeruginosa (2) & $S(2)$ & $S(2)$ & $\mathrm{I}(1) \mathrm{R}(1)$ & $S(1) !(1)$ & $R(2)$ & $\mathrm{R}(2)$ & $R(2)$ & $S(2)$ \\
\hline Pseudomonas putida (1) & $S(1)$ & $S(1)$ & $\mathrm{I}(1)$ & $\mathrm{I}(1)$ & $R(1)$ & $\mathrm{R}(1)$ & $R(1)$ & $S(1)$ \\
\hline Enterobacter sakasakii (1) & $S(1)$ & $S(1)$ & $S(1)$ & $S(1)$ & $R(1)$ & $S(1)$ & $R(1)$ & $S(1)$ \\
\hline Burkholderia pseudomallei (1) & $S(1)$ & $S(1)$ & $\mathrm{R}(1)$ & $\mathrm{I}(1)$ & $R(1)$ & $\mathrm{R}(1)$ & $R(1)$ & $S(1)$ \\
\hline Acinetobacter iwoffi (1) & $S(1)$ & $S(1)$ & $S(1)$ & $S(1)$ & $S(1)$ & $S(1)$ & $S(1)$ & $S(1)$ \\
\hline
\end{tabular}

$\mathrm{S}=$ sensible; $\mathrm{I}=$ intermediate sensibility; $\mathrm{R}=$ resistant

Susceptibilities values $(\mu \mathrm{g} / \mathrm{mL})$ were analyzed according to the Clinical and Laboratory Standards Institute CLSI (2015):

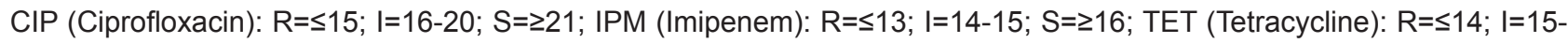
18; $S=\geq 19$; CTX (Cefotaxime): $R=\leq 14 ; \mathrm{I}=15-22 ; \mathrm{S}=\geq 23$; DOX (Doxycycline): $\mathrm{R}=\leq 12 ; \mathrm{I}=13-15 ; \mathrm{S}=\geq 16$; AMC (amoxicillin + clavulanic acid): $R=\leq 13$; I=14-17; $S=\geq 18$; TOB (Tobramycin): $R=\leq 12 ; I=13-14 ; S=\geq 15 ;$ AMO (Amoxicillin): $R=\leq 13$; I=14-16; $S=\geq 17$

the only one Pseudomonas aeruginosa (50\%) showed values of MIC $_{90}$ for doxycycline and cefotaxime greater than $16 \mu \mathrm{g} / \mathrm{mL}$ and $64 \mu \mathrm{g} / \mathrm{mlL}$, respectively (Tables 6 and 7 ).

\section{DISCUSSION}

Enteric bacilli and pseudomonads are opportunistic pathogens at different human body sites. The oral cavity functions as a reservoir for pathogenic species $^{3,17}$. As a result of the aging process, the oral health status declines, often leading to defective oral/denture hygiene in geriatric subjects, being the reasons for this fact the lack of hygiene education of these subjects or of those caring for them. Consequently, oral hygiene is often neglected, resulting in poor oral health and in an increase in the presence of local or general infections that can be related with the presence of enteric microorganisms in the oral cavity ${ }^{4,9}$.

Here we show that dental prostheses can harbor opportunistic pathogens, such as those identified in this study; in addition, these microorganisms can be even resistant to antimicrobial drug therapy as demonstrated here. This is really alarming because, albeit many of these subjects might not be systemically compromised by any disease at the moment they house such pathogens in their oral cavity, they could suffer from any illness that could dampen and compromise their immune system, making them, in turn, more susceptible to be infected by the oral opportunistic pathogens they are harboring.

Thus, a proper denture hygiene protocol is essential, since it could prevent these individuals from exposition to these bacterial species, considering that respiratory pathogens are more capable of colonizing teeth and dentures instead of soft tissues, and pneumonia is the main cause of death related to infection in older people ${ }^{8}$. By wearing dental prostheses, individuals are at a higher risk of aspirating such pathogens from the denture biofilm due to the close proximity of this oral appliance and lungs, being reported a high prevalence of respiratory pathogens on the denture biofilm of hospitalized adults ${ }^{21}$. Furthermore, not only are such pathogens involved in the development of aspiration pneumonia, but also they have been 
Table 6- Percentage of susceptibility of multiresistant microbial species isolated from dental prostheses biofilm regarding Minimum Inhibitory Concentration (MIC) of five antibiotics tested

\begin{tabular}{|c|c|c|c|c|c|}
\hline Microorganism(n) & $\mathrm{CT}^{*}$ & $\mathrm{TM}^{*}$ & $D^{*}$ & IP* & $\mathrm{Cl}^{*}$ \\
\hline \multicolumn{6}{|l|}{ Klebsiella pneumoniae $(\mathrm{n}=9)$} \\
\hline Sensible & 66.6 & 88.9 & 77.8 & 77.8 & 79.8 \\
\hline Intermediate sensibility & 0.0 & 0.0 & $0.0 \%$ & 11.1 & 11.1 \\
\hline Resistent & 33.4 & 11.1 & 22.2 & 11.1 & 11.1 \\
\hline \multicolumn{6}{|l|}{ Escherichia coli $(\mathrm{n}=8)$} \\
\hline Sensible & 87.5 & 87.5 & 37.5 & 100.0 & 100.0 \\
\hline Intermediate sensibility & 12.5 & 0.0 & 25.0 & 0.0 & 0.0 \\
\hline Resistent & 0.0 & 12.5 & 37.5 & 0.0 & 0.0 \\
\hline \multicolumn{6}{|l|}{ Enterobacter aerogenes $(\mathrm{n}=8)$} \\
\hline Sensible & 100.0 & 87.5 & 75.0 & 100.0 & 100.0 \\
\hline Intermediate sensibility & 0.0 & 0.0 & 15.5 & 0.0 & 0.0 \\
\hline Resistent & 0.0 & 12.5 & 12.5 & 0.0 & 0.0 \\
\hline \multicolumn{6}{|l|}{ Citrobacter freundii $(n=4)$} \\
\hline Sensible & 100.0 & 100.0 & 75.0 & 75.0 & 100.0 \\
\hline Intermediate sensibility & 0.0 & 0.0 & 0.0 & 0.0 & 0.0 \\
\hline Resistent & 0.0 & 0.0 & 25.0 & 25.0 & 0.0 \\
\hline \multicolumn{6}{|l|}{ Serratia marcescens $(n=1)$} \\
\hline Sensible & 0.0 & 0.0 & 100.0 & 100.0 & 100.0 \\
\hline Intermediate sensibility & 0.0 & 0.0 & 0.0 & 0.0 & 0.0 \\
\hline Resistent & 100.0 & 100.0 & 0.0 & 0.0 & 0.0 \\
\hline \multicolumn{6}{|l|}{ Enterobacter cloacae $(\mathrm{n}=3)$} \\
\hline Sensible & 100.0 & 100.0 & 66.7 & 100.0 & 100.0 \\
\hline Intermediate sensibility & 0.0 & 0.0 & 0.0 & 0.0 & 0.0 \\
\hline Resistent & 0.0 & 0.0 & 33.3 & 0.0 & 0.0 \\
\hline \multicolumn{6}{|l|}{ Serratia liquefaciens $(n=2)$} \\
\hline Sensible & 100.0 & 100.0 & 0.0 & 50.0 & 100.0 \\
\hline Intermediate sensibility & 0.0 & 0.0 & 0.0 & 0.0 & 0.0 \\
\hline Resistent & 0.0 & 0.0 & 100.0 & 50.0 & 0.0 \\
\hline \multicolumn{6}{|l|}{ Enterobacter gergoviae $(\mathrm{n}=1)$} \\
\hline Sensible & 0.0 & 100.0 & 0.0 & 100.0 & 100.0 \\
\hline Intermediate sensibility & 0.0 & 0.0 & 100.0 & 0.0 & 0.0 \\
\hline Resistent & 100.0 & 0.0 & 0.0 & 0.0 & 0.0 \\
\hline \multicolumn{6}{|l|}{ Pseudomonas aeruginosa $(\mathrm{n}=2)$} \\
\hline Sensible & 50.0 & 100.0 & 50.0 & 100.0 & 100.0 \\
\hline Intermediate sensibility & 0.0 & 0.0 & 50.0 & 0.0 & 0.0 \\
\hline Resistent & 50.0 & 0.0 & 0.0 & 0.0 & 0.0 \\
\hline \multicolumn{6}{|l|}{ Pseudomonas putida $(\mathrm{n}=1)$} \\
\hline Sensible & 100.0 & 100.0 & 100.0 & 100.0 & 100.0 \\
\hline Intermediate sensibility & 0.0 & 0.0 & 0.0 & 0.0 & 0.0 \\
\hline Resistent & 0.0 & 0.0 & 0.0 & 0.0 & 0.0 \\
\hline \multicolumn{6}{|l|}{ Burkholderia pseudomallei $(\mathrm{n}=1)$} \\
\hline Sensible & 100.0 & 100.0 & 100.0 & 100.0 & 100.0 \\
\hline Intermediate sensibility & 0.0 & 0.0 & 0.0 & 0.0 & 0.0 \\
\hline Resistent & 0.0 & 0.0 & 0.0 & 0.0 & 0.0 \\
\hline
\end{tabular}

* CT (0.002-32 $\mu \mathrm{g} / \mathrm{mL}), \mathrm{TM}(0.016-256 \mu \mathrm{g} / \mathrm{mL}), \mathrm{DC}(0.016-256 \mu \mathrm{g} / \mathrm{mL}), \mathrm{IP}(0.002-32 \mu \mathrm{g} / \mathrm{mL}), \mathrm{Cl}(0.002-32 \mu \mathrm{g} / \mathrm{mL})$; Susceptibilities values $(\mu \mathrm{g} / \mathrm{mL})$ were analyzed according to the Clinical and Laboratory Standards Institute CLSI (2015): Cefotaxime (CT) $S \leq 8,16 \leq \mathrm{I} \leq 32$, R $\geq 64$; Tobramycin (TM) $S \leq 4, I=8, R \geq 16$; Doxicyclin (DC) $S \leq 4, I=8, R \geq 16$; Imipenem (IP) $\mathrm{S} \leq 4, \mathrm{I}=8, \mathrm{R} \geq 16$; Ciprofloxacin (Cl) $\mathrm{S} \leq 1, \mathrm{I}=2, \mathrm{R} \geq 4$ 
directly associated with bacterial endocarditis, gastrointestinal infection, and chronic obstructive pulmonary disease. This scenario shows us that daily removal of the denture biofilm is important to prevent the occurrence of associated oral and systemic diseases ${ }^{10}$.

Regarding the prevalence of the aforementioned microorganisms, we found a high prevalence (65.4\%) of Enterobacteriaceae and Pseudomonadaceae species in the denture biofilm from individuals living

Table 7- Minimum Inhibitory Concentration (MIC) values of five antibiotics* tested for multiresistant microbial species isolated from dental prostheses biofilm

\begin{tabular}{|c|c|c|c|c|c|}
\hline Microorganism & $\mathrm{CT}^{\star *}$ & $\mathrm{TM}^{\star \star}$ & $D^{* *}$ & $I^{* * *}$ & $\mathrm{Cl}^{\star *}$ \\
\hline Klebsiella pneumoniae & $>32$ & 12 & 0.19 & 0.75 & 0.25 \\
\hline Klebsiella pneumoniae & 0.094 & 2 & 2 & $>32$ & 0.047 \\
\hline Klebsiella pneumoniae & 0.5 & 2 & 2 & 0.38 & 0.032 \\
\hline Klebsiella pneumoniae & 0.047 & 3 & 2 & 0.25 & 0.064 \\
\hline Klebsiella pneumoniae & 0.064 & 3 & 2 & 0.25 & 0.064 \\
\hline Klebsiella pneumoniae & 0.032 & 3 & 48 & 0.19 & 0.047 \\
\hline Klebsiella pneumoniae & $>32$ & 3 & 16 & 6 & 3 \\
\hline Klebsiella pneumoniae & $>32$ & 3 & 2 & 0.25 & 12 \\
\hline Klebsiella pneumoniae & 0.032 & 3 & 2 & 0.25 & 0.064 \\
\hline Escherichia coli & 0.38 & 16 & 6 & 0.38 & 0.064 \\
\hline Escherichia coli & 0.25 & 1.5 & 3 & 0.38 & 0.064 \\
\hline Escherichia coli & 0.125 & 3 & 12 & 0.25 & 0.023 \\
\hline Escherichia coli & 12 & 0.75 & 32 & 1.5 & 0.125 \\
\hline Escherichia coli & 0.047 & 2 & 1.5 & 0.125 & 0.023 \\
\hline Escherichia coli & 0.064 & 3 & 6 & 0.19 & 0.094 \\
\hline Escherichia coli & 0.125 & 2 & 0.5 & 0.125 & 0.016 \\
\hline Escherichia coli & 2 & 2 & 12 & 0.19 & 0.047 \\
\hline Enterobacter aerogenes & 0.25 & 24 & 6 & 0.38 & 1 \\
\hline Enterobacter aerogenes & 0.25 & 2 & 3 & 0.38 & 0.064 \\
\hline Enterobacter aerogenes & 0.032 & 3 & 3 & 0.25 & 0.047 \\
\hline Enterobacter aerogenes & 0.25 & 3 & $>256$ & 0.38 & 0.064 \\
\hline Enterobacter aerogenes & 0.047 & 2 & 2 & 0.19 & 0.047 \\
\hline Enterobacter aerogenes & 0.016 & 0.75 & 1.5 & 3 & 0.064 \\
\hline Enterobacter aerogenes & 8 & 2 & 2 & 0.25 & 0.064 \\
\hline Enterobacter aerogenes & 0.19 & 3 & 2 & 0.25 & 0.047 \\
\hline Citrobacter freundii & 0.25 & 3 & $>256.0$ & 0.25 & 0.032 \\
\hline Citrobacter freundii & 0.25 & 3 & 3 & $>32$ & 0.047 \\
\hline Citrobacter freundii & 0.094 & 2 & 3 & 0.25 & 0.047 \\
\hline Citrobacter freundii & 0.25 & 3 & 3 & 0.25 & 0.047 \\
\hline Serratia marcescens & $>32$ & 64 & 0.5 & 0.5 & 0.75 \\
\hline Enterobacter cloacae & 0.064 & 0.75 & 3 & 0.19 & 0.012 \\
\hline Enterobacter cloacae & 3 & 0.75 & 24 & 0.19 & 0.032 \\
\hline Enterobacter cloacae & 0.38 & 2 & 3 & 0.38 & 0.023 \\
\hline Serratia liquefaciens & 3 & 0.5 & 16 & 0.38 & 0.047 \\
\hline Serratia liquefaciens & 8 & 2 & $>256$ & $>32$ & 0.5 \\
\hline Enterobacter gergoviae & $>32$ & 1 & 6 & 0.5 & 0.047 \\
\hline Pseudomonas aeruginosa & $>32$ & 1 & 8 & 0.38 & 0.064 \\
\hline Pseudomonas aeruginosa & 8 & 1 & 1.5 & 1.5 & 0.047 \\
\hline Pseudomonas putida & 0.5 & 0.023 & 0.25 & 0.032 & 0.047 \\
\hline Burkholderia pseudomallei & 2 & 0.5 & 3 & 0.19 & 0.003 \\
\hline Burkholderia pseudomallei $(n=1)$ & & & & & \\
\hline
\end{tabular}

* CT (0.002-32 $\mu \mathrm{g} / \mathrm{mL}), \mathrm{TM}(0.016-256 \mu \mathrm{g} / \mathrm{mL}), \mathrm{DC}(0.016-256 \mu \mathrm{g} / \mathrm{mL}), \mathrm{IP}(0.002-32 \mu \mathrm{g} / \mathrm{mL}), \mathrm{Cl}(0.002-32 \mu \mathrm{g} / \mathrm{mL})$ ${ }^{* *} \mu \mathrm{g} / \mathrm{mL}$ 
in the city of Sobral. As opposed to a similar study in a Japanese population, in which the prevalence of potential pathogens on dentures was $18 \%$ for $E$. cloacae and $16 \%$ for $K$. pneumoniae, our research found that $K$. pneumoniae was the most prevalent at $26.5 \%$, followed by Escherichia coli and E. aerogenes, placed after $23.5 \%{ }^{28}$. Another study showed that denture plaque in patients with chronic obstructive pulmonary disease was colonized by pathogens of the respiratory tract, including: $E$. coli, Pseudomonas spp., and Klebsiella spp. Over 33\% of the isolated pathogens are part of rod-shaped and gram-negative Enterobacter spp. The isolation of the mentioned bacteria from denture plaque proves that dental prostheses might become a source of infection of the respiratory tract or may exacerbate chronic respiratory diseases ${ }^{25}$.

Contrary to the data we report, another group described a much lower $(20.3 \%)$ prevalence of enterobacteriaceae in the oral cavity of older people from Greece that used dental prostheses ${ }^{14}$, whereas a different group reported that $48 \%$ of edentulous subjects harbored enteric rods in their oral cavity, being $K$. oxytoca, E. cloacae, and K. pneumoniae species more prevalent ${ }^{12}$. According to these authors, the prevalence of such microorganisms in the denture biofilm may be related to the ability of these species to adhere to the polymer of the denture and to aggregate in the presence of ammonium sulfate. This could explain the high prevalence of $K$. pneumoniae reported in our study.

The classic literature shows that the prevalence of these organisms in the oral cavity of systemically healthy individuals can vary among different populations ${ }^{1,26,27}$. Thus, the high prevalence $(65.4 \%)$ we observed in this study could be attributed to disadvantaged health infrastructure and educational issues that could have led to ingestion of contaminated food or water. Also, poor hygiene habits and indiscriminate use of antibiotics may play a role in this high prevalence ${ }^{4,11}$.

In fact, it was observed that the prostheses hygiene is often precarious. Among the difficulties encountered when conducting a properly cleaning, we can include the lack of adequate patient guidance, characteristics of the prostheses, decreased motor ability, and lack of proper products in the market to carry out the correct denture cleaning. The habit of brushing the prostheses with toothpaste and the use of common brushes may not be the best way to achieve efficient cleaning. Another factor is that the prolonged use of the same prostheses for many years might contribute to the colonization process by potential pathogens ${ }^{4}$. It is relevant to highlight that this information shows a major limitation of these studies, since there are many factors that can lead to microbial colonization of dentures. In our study, we found prostheses being worn for 40 years, and most of the subjects wearing contaminated dentures have been doing so for over 10 years.

Regarding the antimicrobial susceptibility of microorganisms, our data show that $86.5 \%$ of anaerobic facultative gram-negative bacilli were resistant to amoxicillin and over half of them had resistance to amoxicillin with clavulanic acid contrary to other studies that showed the association amoxicillin/clavulanic acid being active on less than half of ampicillin or amoxicillin resistant isolates from prostheses biofilm. Resistance to this association was detected in $28.3 \%$ of the targeted microorganisms and it was particularly frequent in $E$. cloacae, genera Klebsiella, Serratia, and Pseudomonas ${ }^{11}$.

The analysis of the MIC proved that the antimicrobial drug with the highest inhibitory activity against the enteric bacilli and pseudomonads was ciprofloxacin $\left(\mathrm{MIC}_{90} \leq 1 \mu \mathrm{g} / \mathrm{mL}\right.$ ). Nonetheless, we observed a decreased susceptibility to this antimicrobial agent in two (22.2\%) strains of $K$. pneumoniae showing MIC values of 3 and $12 \mu \mathrm{g} / \mathrm{mL}$, respectively. Although it is a much lower frequency than that observed previously ${ }^{20}$, decreased susceptibility rates to ciprofloxacin $\left(\mathrm{MIC}_{90}>2 \mu \mathrm{g} /\right.$ $\mathrm{mL}$ ) among clinical isolates of Enterobacteriaceae species is a particular concern, since enteric rods were able to colonize the denture surface of non-hospitalized subjects, which could spread fluoroquinolones-resistant enterobacteria clones in the community. Fluoroquinolones are the most widely used antibiotics worldwide, and are the drugs of choice for empirical therapy for urinary tract infection. Fluoroquinolone resistance in members of the Enterobacteriaceae family has until recently been attributed to mutations in the gyrase and topoisomerase genes quinolone resistancedetermining regions. Given their transferability and the possibility that they cause increases in resistance that might affect the clinical response to treatment, the detection of quinolone resistance should be routinely performed. There are several surveillance or retrospective studies with clinical isolates, and these studies showed the characteristic features observed in drug-resistant strains in addition to epidemics caused by them ${ }^{33}$.

In this study we also found a slightly higher prevalence (27.5\%) of Enterobacteriaceae species with decreased susceptibility to cefotaxime $\left(\right.$ MIC $_{90} \geq 2 \mu \mathrm{g} / \mathrm{mL}$ ) than the data previously reported of $22.5 \%^{21}$, and these isolates were considered potential ESBL (Extended Spectrum Beta-Lactamases) producers according to the limits established by the CLSI ${ }^{6,7}$. Resistance to $\beta$-lactam antimicrobials in Enterobacteriaceae has been largely due to the presence of $\beta$-lactamase enzymes. $\beta$-lactamase genes were originally found to be chromosomal. $\beta$-lactamase-mediated resistance may develop in vivo during chemotherapy, lending 
support to the prevalent view that ESBL plasmids are conjugative, may be borne on transposons, and that the genes may have high mutation frequencies. Treatment of infections caused by these ESBL-producing bacteria has become challenging. In addition to being resistant to commonly used extended spectrum $\beta$-lactams, these isolates are usually resistant to other classes of antibiotics including fluoroquinolones and aminoglycosides at the same time. One reason for such resistance profile is the practice of irrational usage of antibiotics, leading these microorganisms to exhibit a unique microorganism resistant pattern, which hugely impacts on clinical choice of a correct antibiotic once antimicrobial drug resistance develops, making difficult to treat systemic infections ${ }^{15,22,23}$.

Antimicrobial resistance surveillance programs have provided sufficient data about antimicrobial susceptibility of clinically relevant enteric bacteria and pseudomonads from nosocomial infections and the environment ${ }^{5,18}$. However, they can be found in the biofilm formed on dentures, increasing the risk of the individuals to systemic infections or even worsening them. Thus, it is crucial to emphasize that dentures can harbor such pathogens and this is the reason why a correct hygiene protocol must be carried out in order to avoid such biofilm accumulation on dental prostheses, which, ultimately, would increase the risk of one to be exposed to enteric bacilli.

\section{CONCLUSION}

K. pneumoniae, E. coli, and E. aerogenes were the predominant species found on the denture biofilm. Most enteric bacilli and Pseudomonas spp. were resistant to amoxicillin and amoxicillin clavulanate, with variable susceptibility patterns to other antimicrobial drugs. The antibiotic that showed the highest inhibitory activity against them was ciprofloxacin, but we also found the emergence of ciprofloxacin-reduced sensitivity strains. Therefore, from the results obtained in this study, we suggest that preventative programs for the biofilm control is important to avoid the colonization of dental prostheses by multidrug resistant bacteria as well as avoiding the indiscriminate prescription of antibiotics will help diminish the multidrug resistance insurgence.

\section{ACKNOWLEDGMENTS}

This study was supported by CNPq - National Council for Scientific and Technological Development, Universal Proc. 471158/2007-0, Ministry of Education, and Federative Republic of Brazil.

\section{REFERENCES}

1- Ali RW, Velcescu C, Jivanescu MC, Lofthus B, Skaug N. Prevalence of six putative periodontal pathogens in subgingival plaque samples from Romanian adult periodontitis patients. J Clin Periodontol.1996;23:133-9.

2- An D, Danhorn T, Fuqua C, Parsek MR. Quorum sensing and motility mediate interactions between Pseudomonas aeruginosa and Agrobacterium tumefaciens in biofilm cocultures. Proc Natk Acad Sci U S A. 2006;103:3828-33.

3- Barbosa FC, Irino K, Carbonell GV, Mayer MP. Characterization of Serratia marcescens isolates from subgingival biofilm, extra-oral infections and environment by prodigiosin production, serotyping, and genotyping. Oral Microbiol Immunol. 2006;21:53-60.

4- Berteretche MV, Mastari F, Nicolas E, Hüe O. The needs of denture-brushing in geriatrics: clinical aspects and perspectives. Gerodontology. 2012;29:e768-71.

5- Chen WY, Jang TN, Huang $\mathrm{CH}$, Hsueh PR. In vitro susceptibilities of aerobic and facultative anaerobic Gram-negative bacilli isolated from patients with intra-abdominal infections at a medical center in Taiwan: results of the Study for Monitoring Antimicrobial Resistance Trends (SMART) 2002-2006. J Microbiol Immunol Infect. 2009;42:317-23.

6- Clinical Laboratory Standards Institute - CLSI. Performance standards for antimicrobial disk susceptibility tests. CLSI document. Wayne: CLSI; 2006.

7- Clinical Laboratory Standards Institute - CLSI. Performance standards for antimicrobial susceptibility testing: seventeenth informational supplement. CLSI document MS 100-S25. Wayne: CLSI; 2015.

8- Coulthwaite L, Verran J. Potential pathogenic aspects of denture plaque. Br J Biomed Sci. 2007;64:180-9.

9- Daniluk T, Fiedoruk K, Sciepuk M, Zaremba ML, Rozkiewicz $D$, Cylwik-Rokicka D, et al. Aerobic bacteria in the oral cavity of patients with removable dentures. Adv Med Sci. 2006;51:86-90. 10- Felton D, Cooper L, Duqum I, Minsley G, Guckes A, Haug S, et al. Evidence-based guidelines for the care and maintenance of complete dentures: a publication of the American College of Prosthodontists. J Prosthodont. 2011;20:S1-S12.

11- Gaetti-Jardim EC, Marqueti AC, Faverani LP, Gaetti-Jardim E Jr. Antimicrobial resistance of aerobes and facultative anaerobes isolated from the oral cavity. J Appl Oral Sci. 2010;18:551-9.

12- Goldberg S, Cardash H, Browning H 3rd, Sahly H, Rosenberg M. Isolation of Enterobacteriaceae from mouth and potential association with malodor. J Dent Res. 1997;76:1770-5.

13- Griffin SO, Jones JA, Brunson D, Griffin PM, Bailey WD. Burden of oral disease among older adults and implications for public health priorities. Am J Public Health. 2012;102:411-8.

14- Kaklamanos EG, Charalampidou M, Menexes G, Topitsoglou V, Kalfas S. Transient oral microflora in Greeks attending day centres for the elderly and residents in homes for the elderly. Gerodontology. 2005;22:158-67.

15- Marialouis XA, Santhanam A. Antibiotic resistance, RAPD-PCR typing of multiple drug resistant strains of Escherichia coli from urinary tract infection (UTI). J Clin Diagn Res. 2016;10:5-9.

16- Marsh PD, Percival RS, Challacombe SJ. The influence of denture-wearing and age of the oral microflora. J Dent Res. 1992;71:1374-81.

17- Mathee K, Narasimhan G, Valdes C, Qiu X, Matewish JM, Koehrsen $M$, et al. Dynamics of Pseudomonas aeruginosa genome evolution. Proc Natl Acad Sci U S A. 2008;105:3100-5.

18- Mendes C, Oplustil C, Sakagami E, Turner P, Kiffer C, MYSTIC Brazil Group. Antimicrobial susceptibility in intensive care units: MYSTIC Program Brazil 2002. Braz J Infect Dis. 2005;9:44-51. 19- Meurman JH, Hämäläinen P. Oral health and morbidity implications of oral infections on the elderly. Gerodontology. 2006;23:3-16.

20- Minarini LA, Darini AL. Mutations in the quinolone resistancedetermining region of gyrA and parC in Enterobacteriaceae isolates from Brazil. Braz J Microbiol. 2012;43:1309-14. 
21- O'Donnell LE, Smith K, Williams C, Nile CJ, Lappin DF, Bradshaw $D$, et al. Dentures are a reservoir for respiratory pathogens. J Prosthodont. 2016;25:99-104.

22- Oduro-Mensah D, Obeng-Nkrumah N, Bonney EY, OduroMensah E, Twum-Danso K, Osei YD, et al. Genetic characterization of TEM-type ESBL-associated antibacterial resistance in Enterobacteriaceae in a tertiary hospital in Ghana. Ann Clin Microbiol Antimicrob. 2016:4;15:29.

23- Patzer JA, Dzierzanowska D, Turner PJ. Trends in antimicrobial susceptibility of Gram-negative isolates from a paediatric intensive care unit in Warsaw: results from the MYSTIC programme (19972007). J Antimicrob Chemother. 2008;62:369-75.

24- Przybyłowska D, Mierzwińska-Nastalska E, SwobodaKopeć E, Rubinsztajn R, Chazan R. Potential respiratory pathogens colonisation of the denture plaque of patients with chronic obstructive pulmonary disease. Gerodontology. 2014. doi:10.1111/ger.12156.

25- Ramos MM, Gaetti-Jardim EC, Gaetti-Jardim Junior E. Resistance to tetracycline and $\beta$-lactams and distribution of resistance markers in enteric microorganisms and pseudomonas isolated from the oral cavity. J Appl Oral Sci. 2009;17(Suppl):13-8. 26- Slots J, Feik D, Rams TE. Prevalence and antimicrobial susceptibility of Enterobacteriaceae, Pseudomonadaceae and Acinetobacter in human periodontitis. Oral Microbiol Immunol.1990;5:149-54.

27- Slots J, Rams TE, Feik D, Taveras HD, Gillespie GM. Subgingival microflora of advanced periodontitis in the Dominican Republic. J Periodontol. 1991;62:543-7.
28- Sumi H, Miura M, Sunakawa Y, Michiwak N, Sakagami Y. Colonization of denture plaque by respiratory pathogens in dependent elderly. Gerodontology. 2002;19:25-9.

29- Sumi $Y$, Kagami H, Ohtsuka $Y$, Kakinok $Y$, Haruguchi $Y$, Miyamoto $\mathrm{H}$. High correlation between the bacterial species in denture plaque and pharyngeal microflora. Gerodontology. 2003;20:84-7.

30- Tzouvelekis LS. Markogiannakis A, Psichogiou M, Tassios PT, Daikos GL. Carbapenemases in Klebsiella pneumoniae and other Enterobacteriaceae: an evolving crisis of global dimensions. Clin Microbiol Rev. 2012;25:682-707.

31- United Nations Population Fund - UNPF. Ageing in the twentyfirst century: a celebration and a challenge. New York: UNPF/Help Age International; 2012.

32- Valentini F, Luz MS, Boscato N, Pereira-Cenci T. Biofilm formation on denture liners in a randomised controlled in situ trial. J Dent. 2013;41:420-7.

33- Yamasaki E, Yamada C, Jin X, Nair GB, Kurazono H, Yamamoto $\mathrm{S}$. Expression of marA is remarkably increased from the early stage of development of fluoroquinolone-resistance in uropathogenic Escherichia coli. J Infect Chemother. 2015;21:105-9. 